\title{
An analytical expression for the BER of optimal single user detection of a BPSK signal contaminated by multiple CCls
}

\author{
Kyuhyuk Chung
}

\begin{abstract}
We derive an analytical expression for the bit-error rate (BER) of optimal single user detection of a binary phaseshift keying signal corrupted by multiple cochannel interferers. The channel capacity is also calculated to investigate the BER performance.
\end{abstract}

Keywords: multiple cochannel interference, channel capacity, error probability, maximum likelihood detection

\section{Introduction}

The problem of detecting a binary phase-shift keying (BPSK) signal corrupted by a single cochannel interferer (SCI) and additive white Gaussian noise (AWGN) has been investigated [1-11]. In [1], a suboptimal receiver is derived that utilizes the carrier frequency difference. In [2], an optimal BPSK receiver is derived assuming Rayleigh fading and no receiver knowledge of signal parameters. In [3], a suboptimal BPSK receiver structure is proposed for a non-faded channel. In [4], the optimum receiver is derived for a two-user synchronous BPSK channel. The bit-error rate (BER) performance of the optimum receiver was compared with that of the conventional matched-filter receiver in [4] and the jointly optimal receiver (JOR) in [5]. The exact probability of error of an SCI-JOR was first obtained in $[6,7]$. An exact expression for the BER of an individually optimal receiver (IOR) used to detect a BPSK signal corrupted by a similar SCI and AWGN was derived in [10]. When a BPSK signal corrupted by an SCI and AWGN is detected, the IOR is the optimal multiuser detector [8]. The JOR is also analyzed in [9]. On the other hand, the optimal single user detection (OSUD) in an SCI and AWGN is investigated and the BER of the OSUD is calculated in [11]. However, the number of cochannel interferers (CCIs) can increase for multiuser communications such as cellular mobile systems, in which the domain degradation is the interference due to other users

Correspondence: khchung@dankook.ac.kr

Dankook University, Natural Science Building 501 ho, 126 Jukjeon-dong, Sujigu, Yongin-si, Gyeonggi-do 448-701 Republic of Korea communicating on the same channel, as the number of users increases. In this article, we propose the OSUD for a BPSK signal detection in the presence of AWGN and multiple cochannel interferers (MCIs). In addition, while in [11], the real roots were obtained by the equation of the product tanh functions, we solve the equation specifically, which is obtained by equating log-likelihood ratios (LLRs) with zero. The channel capacity is also calculated to investigate the BER performance.

\section{Signal model and $\mathrm{MCI}-O S U D$ derivation}

We consider an MCI model. Assume that the baseband received signal is given by

$$
r(t)=A_{0} b_{0} s_{0}(t)+\sum_{i=1}^{N_{I}} A_{i} b_{i} s_{i}(t)+n(t)
$$

where $b_{i}, A_{i}$, and $s_{i}(t), i=0,1, \ldots, N_{I}$, are the information bit, amplitude, and signal waveform of the $i$ th user, respectively, the number of interferers is $N_{I}, n(t)$ is an AWGN noise with zero mean and double-sided power spectral density $\sigma^{2}=N_{0} / 2$, and $A_{0} b_{0} s_{0}(t)$ is the desired user's transmitted signal. The cross correlations are defined as $\rho_{i} \triangleq \int_{0}^{T} s_{0}(t) s_{i}(t) d t, i=0,1, \ldots, N_{I}$, where $T$ is the symbol duration. Similar to [10], we assume zero timing error, zero intersymbol interference (ISI), and unit energy for signals. Then, the sampled output of the receiver filter matched to $s_{0}(t)$ is given by 


$$
r_{0}=b_{0} A_{0}+\sum_{i=1}^{N_{I}} b_{i} A_{i} \rho_{i}+n_{0}=b_{0} A_{0}+\sum_{i=1}^{N_{I}} b_{i} I_{i}+n_{0}
$$

where $I_{i}=A_{i} \rho_{i}, i=0,1, \ldots, N_{I}$, and $n_{0}$ is the component of $n(t)$ along $s_{0}(t)$, which is also a Gaussian random variable (RV) with zero mean and variance $\sigma^{2}=N_{0} / 2$. Now, we consider the observation noise which is defined as $w \triangleq \sum_{i=1}^{N_{I}} b_{i} I_{i}+n_{0}$. If binary bits are transmitted with equal probability and independent of each other and also independent of the RV $n_{0}$, then the probability density function (PDF) of $w$ can be calculated as follows

$$
f_{W}(w)=\frac{1}{2^{N_{I}}} \sum_{d=0}^{2^{N_{I}}-1} \mathcal{N}\left(w+\sum_{i=1}^{N_{I}}(-1)^{d_{(2)}\left(N_{I}+1-i\right)} I_{i}\right),
$$

where $d_{(2)}(k)$ is defined as the $k$ th bit in the binary representation of an integer $d$, i.e.,

$$
d \triangleq \sum_{k=1}^{N_{I}} d_{(2)}(k) 2^{k-1} \triangleq\left(d_{(2)}\left(N_{I}\right) \cdots d_{(2)}(2) d_{(2)}(1)\right)_{\left\{2, N_{I}\right\}^{\prime}}
$$

$(\bullet)_{\left\{2, N_{I}\right\}}$ denotes a binary number with $N_{I}$ bits, $d_{(2)}(k)$ $\in\{0,1\}, \mathcal{N}(x)$ is given by $\exp \left(-x^{2} / N_{0}\right) / \sqrt{\pi N_{0}}$. The optimum decision for the desired user's information bit can be obtained from the following hypothesis testing problem,

$$
H_{1}: r_{0}=A_{0}+w, \quad H_{0}: r_{0}=-A_{0}+w .
$$

Using LLRs, the binary decision is given by

$$
\hat{b}_{0}=\operatorname{sgn}\left(\Lambda\left(r_{0}\right)\right)=\operatorname{sgn}\left(\log \frac{f_{R_{0}}\left(r_{0} \mid H_{1}\right)}{f_{R_{0}}\left(r_{0} \mid H_{0}\right)}\right)
$$

where the LLR $\Lambda\left(r_{0}\right)$ is defined as $\log \left(f_{R_{0}}\left(r_{0} \mid H_{1}\right) / f_{R_{0}}\left(r_{0} \mid H_{0}\right)\right)$ and $\operatorname{sgn}(\cdot)$ denotes signum function. Substituting (3) in (5) and after some algebraic manipulation, the OSUD in the presence of AWGN and MCIs is given by

$$
\hat{b}_{0}=\operatorname{sgn}\left[\frac{4 A_{0}}{N_{0}} r_{0}-\Omega\left(r_{0}\right)\right]
$$

where $\Omega\left(r_{0}\right)$ is defined as

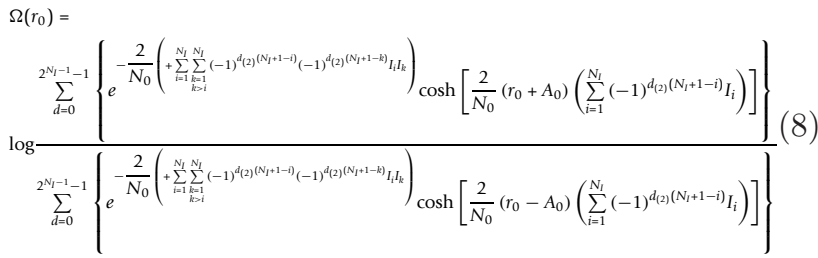

It is easy to show that the MCI-OSUD of (7) simplifies to the SCI-OSUD in [4] for $I_{1} \neq 0$ and $I_{k}=0, k=2,3, \ldots$, $N_{I}$.

\section{BER derivation and channel capacity calculation}

In order to evaluate the BER, we need to find the intervals for $\Lambda\left(r_{0}\right)>0$ or $\Lambda\left(r_{0}\right)<0$. We observe that the numerator and the denominator of the argument of the logarithm in $\Lambda\left(r_{0}\right)$ are the sums of the bell-shaped curves, respectively. The centers of bell curves are numbered as

$$
\begin{gathered}
d+1 \triangleq \mu\left(A_{0}-\sum_{i=1}^{N_{I}}(-1)^{d_{(2)}\left(N_{I}+1-i\right)} I_{i}\right) \\
-c-1 \triangleq \mu\left(-A_{0}+\sum_{i=1}^{N_{I}}(-1)^{c_{(2)}\left(N_{I}+1-i\right)} I_{i}\right)
\end{gathered}
$$

where $d, c=0,1, \ldots, 2^{N_{I}}-1$. Since $\Lambda\left(r_{0}\right)$ is an odd function, the equation of $\Lambda\left(r_{0}\right)=0$ always has a real root $r_{0}=0$. It is very difficult to obtain the exact real roots except a real root $r_{0}=0$. Therefore, we use the Jacobian logarithm to obtain the approximate real roots as follows,

$$
\begin{aligned}
\log \left(e^{x}+e^{y}\right) & =\max (x, y)+\log \left(1+e^{-|y-x|}\right) \\
& \approx \max (x, y) .
\end{aligned}
$$

Then $\Lambda\left(r_{0}\right)=0$ can be written as

$$
\min _{d}\left|r_{0}-\mu^{-1}(d+1)\right| \approx \min _{c}\left|r_{0}-\mu^{-1}(-c-1)\right|,
$$

where $d, c=0,1, \ldots, 2^{N_{I}}-1$. In order to solve (11), we have the case $C_{i}, 1 \leq i \leq N_{c}$, which is a set of marginal conditions, where $N_{c}$ is the number of cases. Then the set $R_{i}$ of real roots corresponding to $C_{i}$ can be obtained by solving (11). We order $2 N_{r}^{(i)}+1$ real roots $p_{j}(i) \in R_{i}$, $j=-N_{r}^{(i)},-N_{r}^{(i)}+1, \ldots,-1,0,1, \ldots, N_{r}^{(i)}-1, N_{r}^{(i)}, \quad$ as follows,

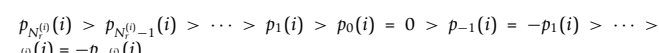

Then, given the case $C_{i}$, the decision intervals for $\Lambda\left(r_{0}\right)>0$ are given by

$$
\begin{aligned}
& \begin{array}{l}
p_{N_{r}^{(i)}}(i)<r_{0} \\
p_{N_{r}^{(i)}-2}(i)<r_{0}<p_{N_{r}^{(i)}-1}(i)
\end{array} \\
& -p_{N_{r}^{(i)}-2}(i)<r_{0}<-p_{N_{r}^{(i)}-3}(i) \\
& -p_{N_{r}^{(i)}}(i)<r_{0}<-p_{N_{r}^{(i)}-1}(i)
\end{aligned}
$$

For the case $C_{1}$, the BER $P_{b}^{(1)}$ is calculated as follows

$$
P_{b}^{(1)}=\frac{1}{2^{N_{I}}} \sum_{d=0}^{2^{N_{I}}-1} Q\left(\frac{\mu^{-1}(d+1)}{\sqrt{N_{0} / 2}}\right) .
$$


For the case $C_{i}, 2 \leq i \leq N_{c}$, and the center $-c-1$, $-2^{N_{I}} \leq-c-1 \leq-1$, we can obtain the following condition

$$
p_{j-2}(i)<p_{j-1}(i)<\mu^{-1}(-c-1)<p_{j}(i)<p_{j+1}(i)
$$

Then the conditional BER is calculated as follows

$$
P_{-c-1}^{(i)} \approx \sum_{h=0}^{N^{(i)-j}} \frac{(-1)^{h}}{2^{N_{t}}} Q\left(\frac{p_{i+h}(i)-\mu^{-1}(-c-1)}{\sqrt{N_{0} / 2}}\right)+\sum_{h=0}^{N^{(i) j-1}} \frac{(-1)^{h}}{2^{N_{N}}} Q\left(\frac{\mu^{-1}(-c-1)-p_{j-1-h}(i)}{\sqrt{N_{0} / 2}}\right),
$$

where $Q(x)=\int_{x}^{\infty}(1 / \sqrt{2 \pi}) \exp \left(-t^{2} / 2\right) d t$. Then the $\operatorname{BER} P_{b}^{(i)}$ is calculated as follows

$$
P_{b}^{(i)} \approx \sum_{c=0}^{2^{N_{I}}-1} P_{-c-1}^{(i)}
$$

where $2 \leq i \leq N_{c}$.

\subsection{BER derivation for $N_{l}=2$}

We continue our derivation for $N_{I}=2$ and without loss of generality, we assume $I_{1} \geq I_{2}$. Then we can obtain the real roots solving (11) as follows

$$
\begin{array}{ll}
0 & \text { for } A_{0}>I_{1}+I_{2}, \\
0, \pm I_{2} & \text { for } A_{0}<I_{1}+I_{2}, A_{0}>I_{1}, \\
0, \pm I_{2}, \pm I_{1} & \text { for } A_{0}>I_{1}-I_{2}, I_{1}>A_{0}>I_{2} \\
0, \pm I_{1} & \text { for } A_{0}<I_{1}-I_{2}, I_{1}>A_{0}>I_{2} \\
0, \pm I_{1}, \pm\left(I_{1}+I_{2}\right) & \text { for } A_{0}>I_{1}-I_{2}, I_{1}>I_{2}>A_{0} \\
0, \pm I_{1}, \pm\left(I_{1}+I_{2}\right), \pm\left(I_{1}-I_{2}\right) & \text { for } A_{0}<I_{1}-I_{2}, I_{1}>I_{2}>A_{0} .
\end{array}
$$

Then, the BER can be calculated by (14) or (17).

\section{$2^{\prime \prime}, 1,0,1,0,0 p c, 0 p c, 0 p c, 0 p c>3.2$. BER derivation for $N_{l}>2$}

Next, we continue the derivation for $N_{I}>2$, which is more complicated than the previous case because the number $N_{c}$ of cases increases rapidly. To make the problem tractable, we assume the following condition

$$
A_{0}>I_{1}>2 I_{2}>\cdots>2^{N_{\mathrm{I}}-1} I_{N_{\mathrm{I}}}>0
$$

In the above condition, a practical situation, in which the interference of the CCIs is not severe, can be assumed. Then the possible marginal conditions for various real roots are given by

$$
\begin{aligned}
& \mu^{-1}(-c-1)-\mu^{-1}(d+1)<0 \\
& -\mu^{-1}(-c-1)+\mu^{-1}(d+1)<0
\end{aligned}
$$

where $d, c=0,1, \ldots, 2^{N_{I}}-1$. These conditions can be written as greatly simple forms with ternary numbers, which are

$$
t \triangleq \sum_{k=1}^{N_{I}} t_{(3)}(k) 3^{k-1} \triangleq\left(t_{(3)}\left(N_{I}\right) \cdots t_{(3)}(2) t_{(3)}(1)\right)_{\left\{3, N_{I}\right\}^{\prime}}
$$

where $(\bullet)_{\left\{3, N_{I}\right\}}$ denotes a ternary number with $N_{I}$ digits, and $t_{(3)}(k) \in\{0,1,2\}$ is defined as the $k$ th digit in the ternary representation of an integer $t$. The left-hand sides in (20) are numbered as

$$
\begin{gathered}
3^{N_{I}}-t \triangleq \lambda\left(A_{0}-\sum_{i=1}^{N_{I}}\left(t_{(3)}\left(N_{I}+1-i\right)-1\right) I_{i}\right), \\
-3^{N_{I}}+t \triangleq \lambda\left(-A_{0}+\sum_{i=1}^{N_{I}}\left(t_{(3)}\left(N_{I}+1-i\right)-1\right) I_{i}\right),
\end{gathered}
$$

where $t=0,1, \ldots, 3^{N_{I}}-1$. The conditions for various real roots can efficiently be represented as a table. Now, we explain the procedure to create the table. The first step is to draw a table with $(11 \cdots 1)_{\left\{3, N_{I}-2\right\}}$ rows and $(11 \cdots 1)_{\left\{3, N_{I}-1\right\}}$ columns. Fill the first row with the decimal numbers from left to right, starting with 1 and ending with $(11 \cdots 1)_{\left\{3, N_{I}-1\right\}}$. Then copy the one-cell left-shifted version of the first row into the second row, the onecell left-shifted version of the second row into the third row, and so on. Remove the entries below the main diagonal. On the first row, find the numbers for inequalities corresponding to $\mu^{-1}(d+1)<\mu^{-1}(-c-1)$, where $d=0,1, \ldots, 2^{N_{I}-1}-1$ and $c=0$. Write $-c-1$ on a new additional column, following the same pattern as $d+1$ with the opposite sign, where $c=0,1, \ldots, 2^{N_{I}-2}-1$. Finally, mark the entries at the intersection of $-c-1$ rows and $d+1$ columns. Figure 1 shows the procedure for generating the table with $N_{I}$ $=4$ interferers. The number of columns is the number of conditions for various real roots, which are numbered as 1 to $1+(11 \cdots 1)_{\left\{3, N_{I}-1\right\}}$. Then the set $C_{i}$ with $N_{c}=1+(11 \cdots 1)_{\left\{3, N_{I}-1\right\}}$ is defined as

$$
C_{i}=\left\{\lambda^{-1}(-m(-c-1))<0, \lambda^{-1}(n(-c-1))<0 \mid c=0,1, \ldots, 2^{N_{l}-2}-1\right\}
$$

where $m(-c-1)$ is the biggest and nearest marked number from $i$ including $i$ and $n(-c-1)$ is the smallest and nearest marked number from $i$ excluding $i$ on each row $-c-1$, where $-2^{N_{I}-2} \leq-c-1 \leq-1$. If $m$ does not exist, there is no corresponding marginal condition. The set $D_{i}$ of candidates for real roots corresponding to the case $C_{i}$ is defined as

$$
\begin{aligned}
& D_{i}=\left\{ \pm\left(\mu^{-1}\left(d_{m(-c-1)}+1\right)-\mu^{-1}(-c-1)\right) / 2\right. \\
& \quad \pm\left(\mu^{-1}(-c-1)-\mu^{-1}\left(d_{n(-c-1)}+1\right)\right) / 2 \\
& \left.0 \mid c=0,1, \ldots, 2^{N_{I}-2}-1\right\}
\end{aligned}
$$

where the indexes $\left(d_{m(-c-1)}+1\right)$ and $\left(d_{n(-c-1)}+1\right)$ of the centers of bell curves correspond to $m(-c-1)$ and $n(-c-1)$ on each row $-c-1$, respectively. For the set $E_{i}$ given by 
$E_{i}=\left\{\left(-c_{1}-1\right),\left(-c_{2}-1\right) \mid\left(d_{m\left(-c_{1}-1\right)}+1\right)=\left(d_{m\left(-c_{2}-1\right)}+1\right)\right.$,

$\left.c_{1}, c_{2}=0,1, \ldots, 2^{N_{l}-2}-1, c_{1} \neq c_{2}\right\}$,

the updated set $F_{i}$ is obtained from the set $D_{i}$ by removing $\pm\left(\mu^{-1}\left(d_{m(-c-1)}+1\right)-\mu^{-1}(-c-1)\right) / 2$, except the biggest element $(-b-1) \in E_{i}$, for $(-c-1) \neq(-b-1),(-c-1) \in E_{i}$, and the updated set $R_{i}$ is obtained from the set $F_{i}$ by removing $\pm\left(\mu^{-1}(-c-1)-\mu^{-1}\left(d_{n(-c-1)}+1\right)\right) / 2$, except the smallest element $(-s-1) \in E_{i}$, for $(-c-1) \in E_{i},(-c-$ $1) \neq(-s-1)$.

\begin{tabular}{|l|l|l|l|l|l|l|l|l|l|l|l|l|l|}
\hline 1 & 2 & 3 & 4 & 5 & 6 & 7 & 8 & 9 & 10 & 11 & 12 & 13 & 14 \\
\hline & & & & & & & & & & & & & \\
\hline & & & & & & & & & & & & & \\
\hline & & & & & & & & & & & & & \\
\hline & & & & & & & & & & & & & \\
\hline
\end{tabular}

\begin{tabular}{|l|l|l|l|r|r|r|r|r|r|r|r|r|r|}
\hline 1 & 2 & 3 & 4 & 5 & 6 & 7 & 8 & 9 & 10 & 11 & 12 & 13 & 14 \\
\hline 2 & 3 & 4 & 5 & 6 & 7 & 8 & 9 & 10 & 11 & 12 & 13 & 14 & \\
\hline 3 & 4 & 5 & 6 & 7 & 8 & 9 & 10 & 11 & 12 & 13 & 14 & & \\
\hline 4 & 5 & 6 & 7 & 8 & 9 & 10 & 11 & 12 & 13 & 14 & & & \\
\hline 5 & 6 & 7 & 8 & 9 & 10 & 11 & 12 & 13 & 14 & & & & \\
\hline
\end{tabular}

\begin{tabular}{|c|c|c|c|c|c|c|c|c|c|c|c|c|c|}
\hline 1 & 2 & 3 & 4 & 5 & 6 & 7 & 8 & 9 & 10 & 11 & 12 & 13 & 14 \\
\hline & 3 & 4 & 5 & 6 & 7 & 8 & 9 & 10 & 11 & 12 & 13 & 14 & \\
\hline & & 5 & 6 & 7 & 8 & 9 & 10 & 11 & 12 & 13 & 14 & & \\
\hline & & & 7 & 8 & 9 & 10 & 11 & 12 & 13 & 14 & & & \\
\hline & & & & 9 & 10 & 11 & 12 & 13 & 14 & & & & \\
\hline
\end{tabular}

\begin{tabular}{|l|}
\hline$-c-1 \backslash d+1$ \\
\hline-1 \\
\hline-2 \\
\hline-3 \\
\hline-4 \\
\hline
\end{tabular}

\begin{tabular}{|l|l|l|l|l|l|l|l|l|l|l|l|l|l|}
\hline+1 & +2 & & +3 & +4 & & & & & +5 & +6 & & +7 & +8 \\
\hline 1 & 2 & 3 & 4 & 5 & 6 & 7 & 8 & 9 & 10 & 11 & 12 & 13 & 14 \\
\hline & 3 & 4 & 5 & 6 & 7 & 8 & 9 & 10 & 11 & 12 & 13 & 14 & \\
\hline & & 5 & 6 & 7 & 8 & 9 & 10 & 11 & 12 & 13 & 14 & & \\
\hline & & & 7 & 8 & 9 & 10 & 11 & 12 & 13 & 14 & & & \\
\hline & & & & 9 & 10 & 11 & 12 & 13 & 14 & & & & \\
\hline
\end{tabular}

\begin{tabular}{|l|}
\hline$-c-1 \backslash d+1$ \\
\hline-1 \\
\hline-2 \\
\hline-3 \\
\hline-4 \\
\hline
\end{tabular}

\begin{tabular}{|l|l|l|l|l|l|l|l|l|l|l|l|l|l|}
\hline+1 & +2 & & +3 & +4 & & & & & +5 & +6 & & +7 & +8 \\
\hline 1 & 2 & 3 & 4 & {$[5$} & 6 & 7 & 8 & 9 & 10 & 11 & 12 & 13 & 14 \\
\hline & 3 & 4 & 5 & 6 & 7 & 8 & 9 & 10 & 11 & 12 & 13 & 14 & \\
\hline & & 5 & 6 & 7 & 8 & 9 & 10 & 11 & 12 & 13 & 14 & & \\
\hline & & & 7 & 8 & 9 & 10 & 11 & 12 & 13 & 14 & & & \\
\hline & & & & 9 & 10 & 11 & 12 & 13 & 14 & & & & \\
\hline
\end{tabular}

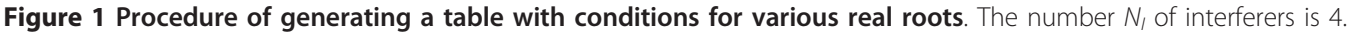




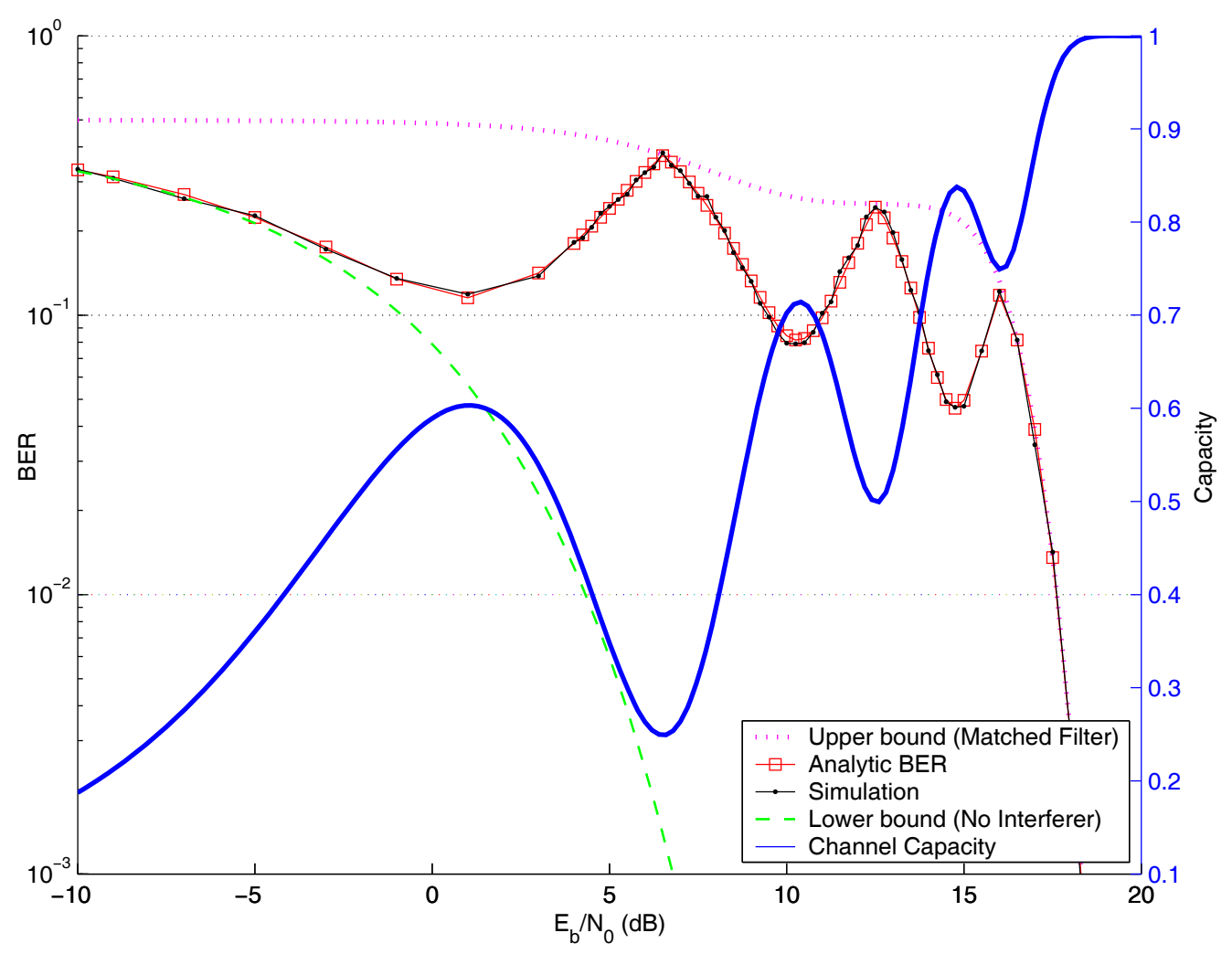

Figure 2 The left axis is the BER and the right axis is the capacity (in bit/channel use) for $I_{1}=6.0$ and $I_{2}=3.0$ with $N_{0}=2.0$.

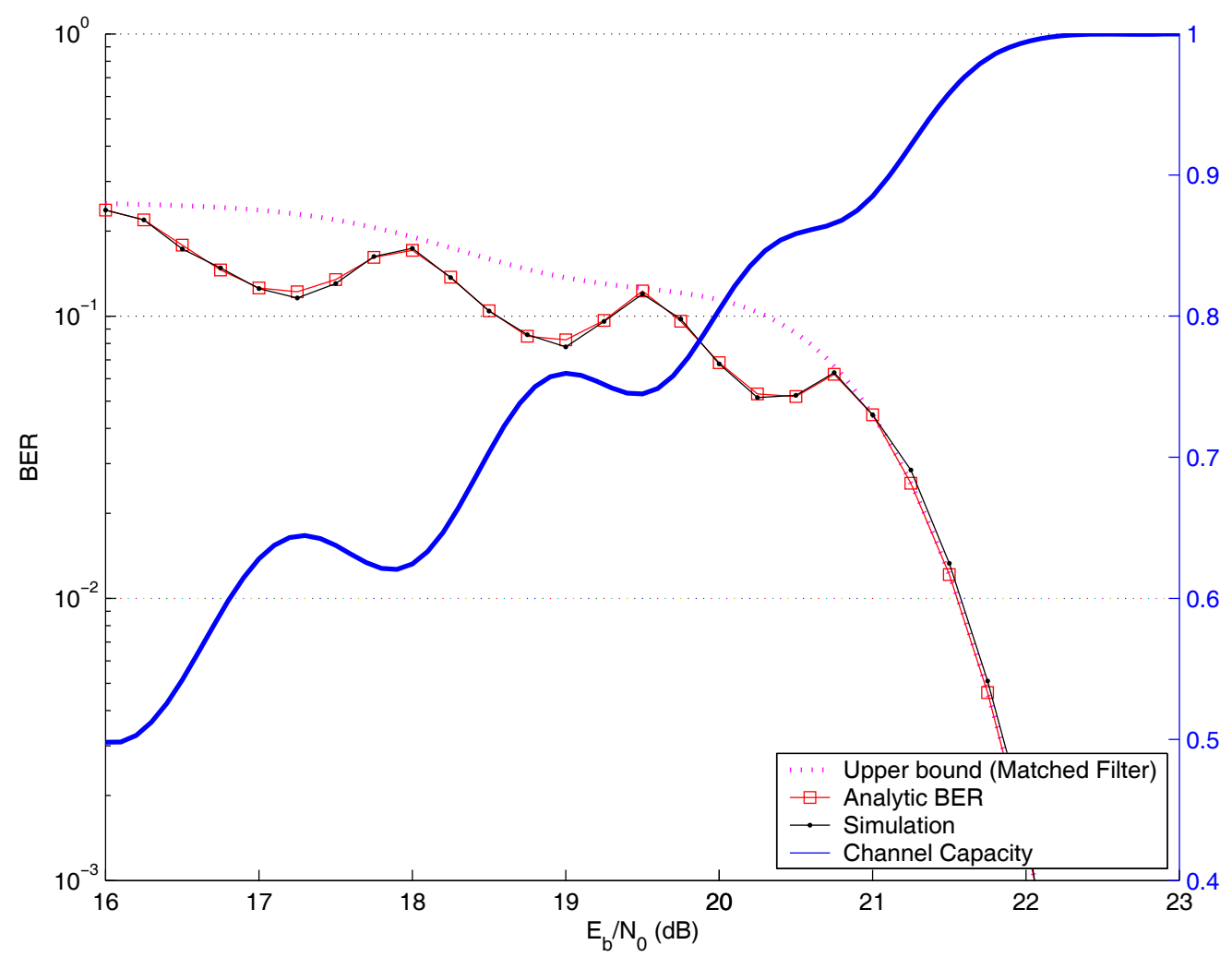

Figure 3 The left axis is the BER and the right axis is the capacity (in bit/channel use) for $I_{1}=9.0, I_{1}=4.4, l_{3}=2.1, I_{4}=1.0$ and with $N_{0}=2.0$. 
Figure 1. Procedure of generating a table with conditions for various real roots. The number $N_{I}$ of interferers is 4 .

In summary, with the set $C_{i}$ of marginal conditions, we obtain the set $R_{i}$ of real roots corresponding to $C_{i}$ by solving (11). For the case $C_{i}, 1 \leq i \leq N_{c}$, the real roots $p_{j}(i)$ $\in R_{i}, \quad j=-N_{r}^{(i)}, \ldots, N_{r}^{(i)}$, and the center $-c-1$, $-2^{N_{I}} \leq-c-1 \leq-1$, we obtain the condition $p_{j-1}(i)<\mu^{-}$ ${ }^{1}(-c-1)<p_{j}(i)$. Then conditional BER $P_{-c-1}^{(i)}$ is calculated by (16) and the BER $P_{b}^{(i)}$ is calculated by (14) or (17).

In addition, we calculate the channel capacity. We consider the channel of (4) with possible inputs $A_{0}$ or $A_{0}$. The capacity of this channel in bit/channel use is given by [12]

$$
\begin{aligned}
C & =1-\frac{1}{2} \int_{-\infty}^{\infty} f_{R_{0}}\left(r_{0} \mid H_{1}\right) \log _{2}\left(1+e^{-\Lambda\left(r_{0}\right)}\right) d r_{0} \\
& -\frac{1}{2} \int_{-\infty}^{\infty} f_{R_{0}}\left(r_{0} \mid H_{0}\right) \log _{2}\left(1+e^{\Lambda\left(r_{0}\right)}\right) d r_{0}
\end{aligned}
$$

where by using (3) and (4), $f_{R_{0}}\left(r_{0} \mid H_{1}\right)=f_{W}\left(r_{0}-A_{0}\right)$ and $f_{R_{0}}\left(r_{0} \mid H_{0}\right)=f_{W}\left(r_{0}+A_{0}\right)$. Then the capacity is computed by (26).

\section{Results}

Figure 2 shows analytical BERs, simulations, and channel capacity of the proposed MCI-OSUD for $N_{I}=2$. We define the signal-to-noise ratio (SNR) as $E_{b} / N_{0} \triangleq A_{0}^{2} / N_{0}$. The upper bound, which is the hard decision for the outputs of the matched filter, is also shown. When there is no interferer, the BER is a lower bound. As the SNR changes, i.e., the amplitude $A_{0}$ of the desired user, the condition $C_{i}$ satisfied also changes even though $I_{k}$ 's are fixed. The analytical BER coincides with the simulation. This validates our approximation. For the low SNR region, the BER performance of the MCI-OSUD approaches that of no interferer case. After fluctuations due to multiple interferers, the MCI-OSUD BER performance approaches that of the matched filter. The channel capacity is also in good agreement with the MCI-

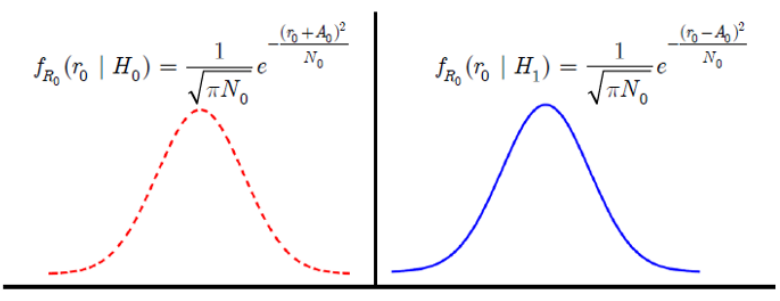

(a)

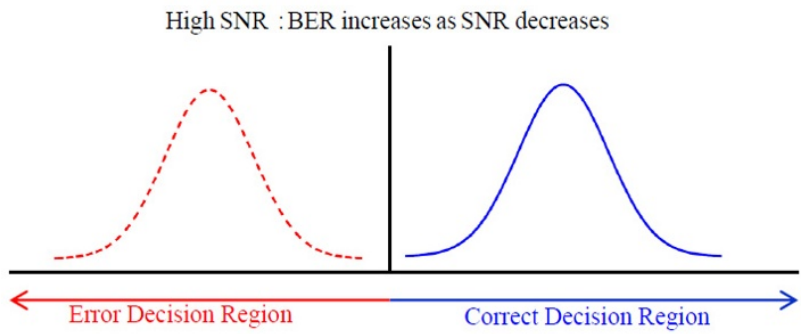

(b)

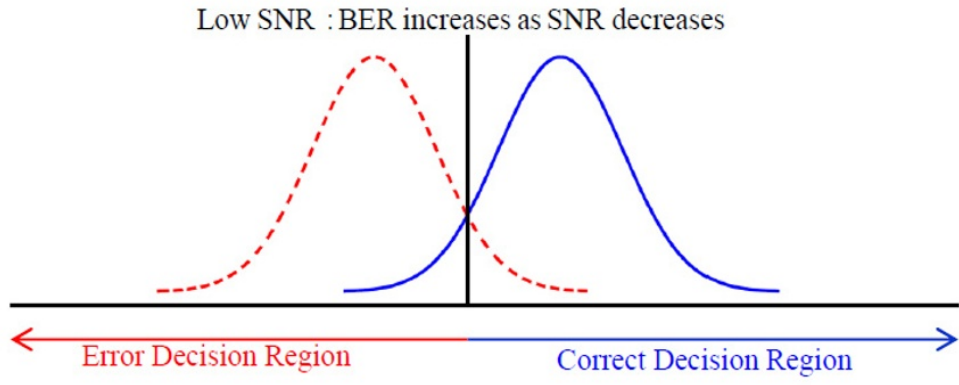

(c)

Figure 4 Illustration of no interferer case 
OSUD BER, i.e., the higher the capacity is, the lower the $B E R$ is and vice versa. We note that while analytical BERs, simulations, and the channel capacity of the SCIOSUD in [11] have a single local minimum, those of the proposed MCI-OSUD have multiple local minima, which show the existence of multiple interferers. Similar results are obtained for $N_{I}=4$, shown in Figure 3. Note that for $N_{I}>2$, we assume (19). Therefore, the leftmost SNR, i.e.,

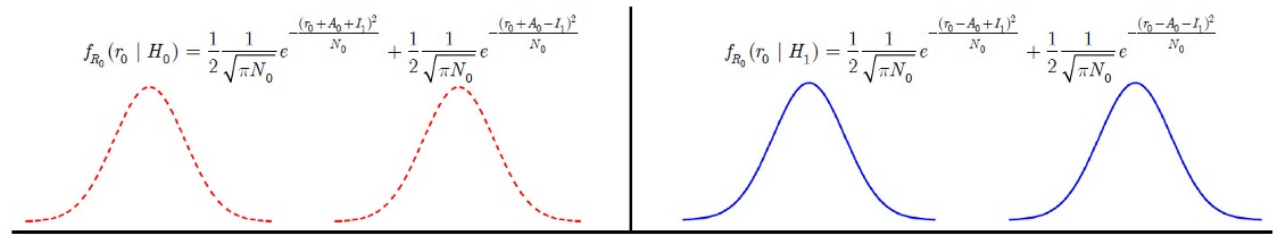

(a)

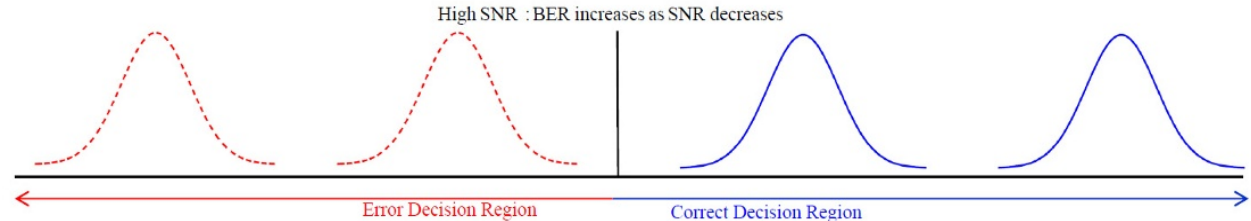

(b)

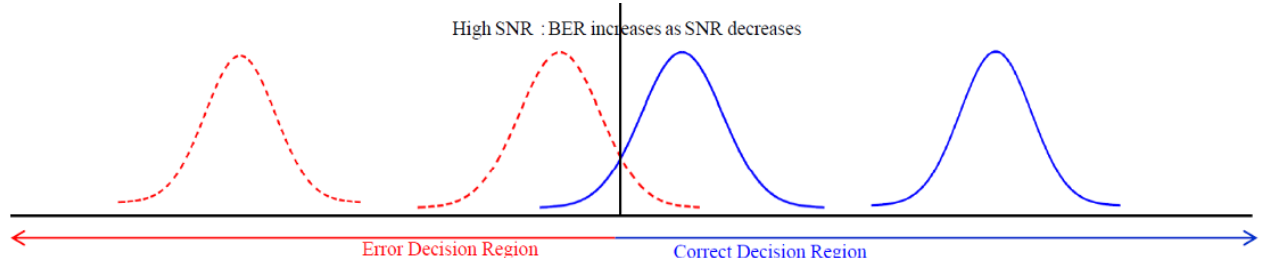

(c)

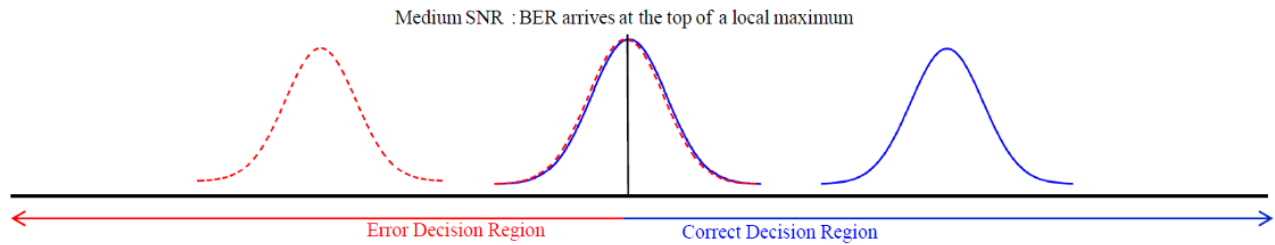

(d)

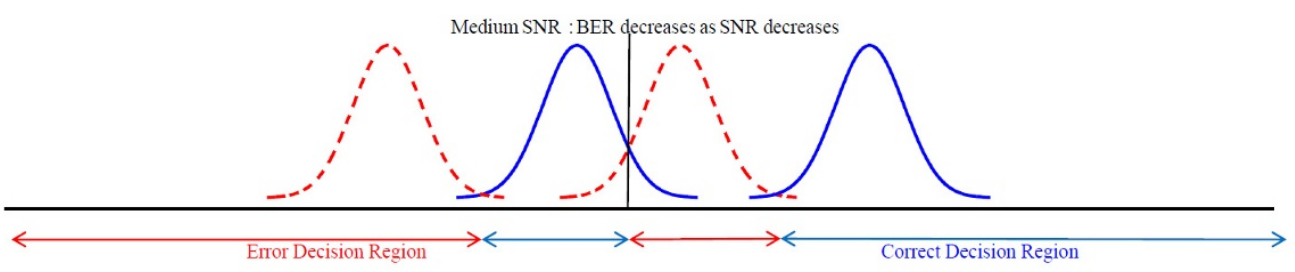

(e)

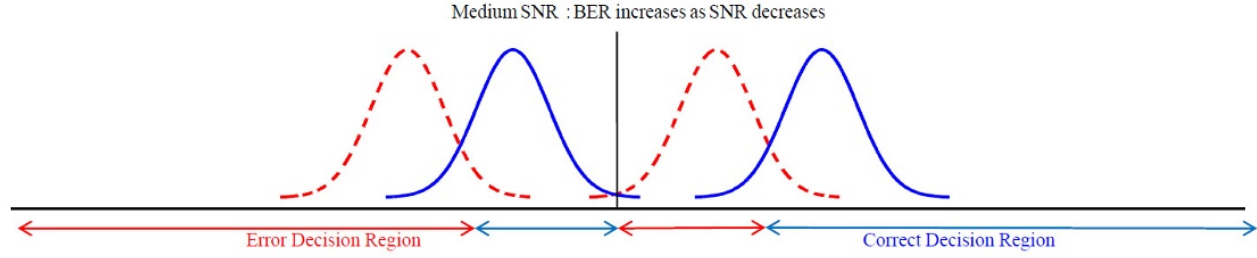

(f)

Figure 5 Illustration of a single interferer case 
$16 \mathrm{~dB}$, in Figure 3 is the minimum SNR, which satisfies (19). Note that the leftmost SNR is not a limitation because we can change $N_{0}$ so that the different leftmost SNR is obtained. For simulations, we used Monte Carlo simulators. Then, we averaged over 50,000 trials at each SNR. In addition, we define the maximum mismatch as the maximum value of absolute differences between the simulations and the analytical results. For the simulations in Figures 2 and 3, we obtained the maximum mismatches of 0.014 and 0.0081 , respectively. Usually, the BER is non-increasing and the capacity is non-decreasing as the SNR increases. However, with CCIs, generally these are not true. This phenomenon can be explained clearly with the simplest case, i.e., a single interferer case. Before we explain a single interferer case, let us first review no interferer case. For this case, the conditional PDFs are given as in Figure 4a. Then at high SNRs, the BER increases as the SNR decreases. The decision region is shown in Figure 4b. For this case, the BER increases monotonically until the SNR reaches at the low SNR as shown in Figure 4c. However, with CCIs, generally the BER does not increase monotonically as the SNR decreases. For this case, the conditional PDFs are given in Figure 5a. Then at high SNRs, the BER increases as the SNR decreases. The decision region is shown in Figure $5 \mathrm{~b}$. The BER increases monotonically until the SNR reaches at some SNR as shown in Figure 5c. Then the BER arrives at the top of a local maximum shown in Figure $5 \mathrm{~d}$. After the BER reaches the local maximum, the BER decreases as the SNR decreases over the medium SNR region shown in Figure 5e. Then after the BER reaches the local minimum, the BER increases as the SNR decreases as usual shown in Figure 5f. As shown above, the physical interpretation is now clear. When there exists CCIs, they can interfere constructively as well as destructively. Therefore, the BER (and in turn the capacity) is not always monotonically increasing or decreasing for the cochannel interference case.

\section{Conclusion}

We derived an analytical expression for the BER of the MCI-OSUD. The effect of MCIs on the BER was analyzed. To investigate the BER performance, the channel capacity was also calculated. The capacity, the analytical result, and the simulation are in good agreement.

\section{Competing interests}

The author declares that they have no competing interests.

Received: 7 January 2012 Accepted: 8 June 2012 Published: 8 June 2012

\section{References}

1. MJ Wilmut, Signal detection in the presence of co-channel interference and noise, (Queen's University, 1970)
2. H Camkerten, Optimum one-shot BPSK receiver design for multiple access mobile radio channels with Rayleigh fading, in Proc IEEE VTC 1992, Denver. 1, 447-450 (May 1992)

3. GJM Janssen, Receiver structure for simultaneous reception of two BPSK modulated cochannel signals. Electron Lett. 29(12), 1095-1097 (1993). doi:10.1049/el:19930731

4. R Kwan, C Leung, Optimal detection of a BPSK signal contaminated by interference and noise. IEEE Commun Lett. 6(2), 225-227 (2002)

5. TV Poon, NC Beaulieu, Jointly and individually optimum receivers for BPSK signals in cochannel interference plus noise, in Proc IEEE Pacific Rim Conf Commun, Comput, Signal Process, Victoria, BC, Canada, 2, 530-532 (August 2003)

6. TV Poon, NC Beaulieu, Performance analysis of a jointly optimal BPSK receiver in cochannel interference, in Proc IEEE Global Telecommn Conf, San Francisco, USA, 3, 1721-1725 (December 2003)

7. TV Poon, NC Beaulieu, Error performance analysis of a jointly optimal single cochannel-interferer BPSK receiver. IEEE Trans Commun. 52(7), 1051-1054 (2004). doi:10.1109/TCOMM.2004.831359

8. S Verdú, Multiuser Detection, 1st edn. (Cambridge University Press, New York, 1998)

9. AM Rabiei, NC Beaulieu, A simple, intuitive expression for the BER of a jointly optimal single cochannel interferer BPSK receiver. IEEE Commun Lett. 9(3), 201-203 (2005). doi:10.1109/LCOMM.2005.03030

10. AM Rabiei, NC Beaulieu, An analytical expression for the BER of an individually optimal single cochannel interferer BPSK receiver. IEEE Trans Commun. 55(1), 60-63 (2007)

11. SM Saberali, H Amindavar, AR Moghaddamjoo, BER calculation and investigation of optimal single user detector for a BPSK signal contaminated by cochannel interferer. IEEE Commun Lett. 12(10), 705-707 (2008)

12. JG Proakis, Digital Communications, 4th edn. (McGraw-Hill, New York, 2001)

doi:10.1186/1687-1499-2012-190

Cite this article as: Chung: An analytical expression for the BER of optimal single user detection of a BPSK signal contaminated by multiple CCIs. EURASIP Journal on Wireless Communications and Networking 2012 2012:190.

\section{Submit your manuscript to a SpringerOpen ${ }^{\mathcal{O}}$ journal and benefit from: \\ - Convenient online submission \\ - Rigorous peer review \\ - Immediate publication on acceptance \\ - Open access: articles freely available online \\ - High visibility within the field \\ - Retaining the copyright to your article}

Submit your next manuscript at $\boldsymbol{s p r i n g e r o p e n . c o m ~}$ 